\title{
Effects of the Angiotensin Receptor Blocker Olmesartan on Adipocyte Hypertrophy and Function in Mice with Metabolic Disorders
}

\author{
Akinobu Maeda, ${ }^{1}$ Kouichi Tamura, ${ }^{1}$ Hiromichi Wakui, ${ }^{1}$ \\ Masato Ohsawa, ${ }^{1}$ Kengo Azushima, ${ }^{1}$ Kazushi Uneda, ${ }^{1}$ Tomohiko Kanaoka, ${ }^{1}$ \\ Ryu Kobayashi, ${ }_{1}^{1}$ Kohji Ohki, ${ }^{1}$ Miyuki Matsuda, ${ }^{1}$ Yuko Tsurumi-Ikeya, ${ }^{1}$ \\ Akio Yamashita, ${ }^{2}$ Yasuo Tokita, ${ }^{3}$ and Satoshi Umemura ${ }^{1}$ \\ ${ }^{1}$ Department of Medical Science and Cardiorenal Medicine, Yokohama City University Graduate School of Medicine, \\ 3-9 Fukuura, Kanazawa-ku, Yokohama 236-0004, Japan \\ ${ }^{2}$ Department of Molecular Biology, Yokohama City University Graduate School of Medicine, 3-9 Fukuura, Kanazawa-ku, \\ Yokohama 236-0004, Japan \\ ${ }^{3}$ Renal Division, Department of Medicine, Fujisawa Municipal Hospital, 2-6-1 Fujisawa, Fujisawa 251-8550, Japan
}

Correspondence should be addressed to Kouichi Tamura; tamukou@med.yokohama-cu.ac.jp

Received 14 March 2014; Revised 10 May 2014; Accepted 10 May 2014; Published 2 June 2014

Academic Editor: Cheng Hu

Copyright (C) 2014 Akinobu Maeda et al. This is an open access article distributed under the Creative Commons Attribution License, which permits unrestricted use, distribution, and reproduction in any medium, provided the original work is properly cited.

In the present study, we examined the therapeutic effects of olmesartan, an angiotensin II (Ang II) type 1 receptor (AT1R)-specific blocker, in genetically obese diabetic KKAy mice, a model of human metabolic disorders with visceral obesity, with a focus on an olmesartan effect on the adipose tissue. Olmesartan treatment $(3 \mathrm{mg} / \mathrm{kg}$ per day) for 4 weeks significantly lowered systolic blood pressure but did not affect body weight during the study period in KKAy mice. However, there were three interesting findings possibly related to the pleiotropic effects of olmesartan on adipose tissue in KKAy mice: (1) an inhibitory effect on adipocyte hypertrophy, (2) a suppressive effect on IL-6 gene expression, and (3) an ameliorating effect on oxidative stress. On the other hand, olmesartan exerted no evident influence on the adipose tissue expression of AT1R-associated protein (ATRAP), which is a molecule interacting with AT1R so as to inhibit pathological AT1R activation and is suggested to be an emerging molecular target in metabolic disorders with visceral obesity. Collectively, these results suggest that the blood pressure lowering effect of olmesartan in KKAy mice is associated with an improvement in adipocyte, including suppression of adipocyte hypertrophy and inhibition of the adipose IL-6-oxidative stress axis. Further study is needed to clarify the functional role of adipose ATRAP in the pleiotropic effects of olmesartan.

\section{Introduction}

Recently, metabolic disorder with visceral obesity has come to be recognized as a major medical condition related to significantly increased risks of hypertension, type 2 diabetes, dyslipidemia, and ultimately life-threatening cardiovascular disease [1]. Accumulating evidence also indicates that adipose tissue functions as a distinct endocrine organ capable of producing adipokines, such as adiponectin and leptin [2]. Furthermore, dysregulation of adipose tissue function is suggested to be closely involved in the pathophysiology of metabolic disorders via the stimulated production of inflammatory cytokines and upregulation of oxidative stress [3-5].

The renin-angiotensin system (RAS) plays an important role in the maintenance of circulatory and water-electrolyte homeostasis based on the generation of angiotensin II (Ang II), a potent vasoactive peptide, and the pathological activation of RAS has been implicated as one of the major contributors to hypertension and cardiovascular disease. Recent evidence has also indicated an important role of adipose tissue RAS in the physiological regulation of adipose tissue 
function, further suggesting a specific pathophysiological link between the dysregulated activation of adipose tissue RAS and the development of metabolic disorders and their complications $[6,7]$. The physiological and pathophysiological actions of Ang II are principally mediated by the Ang II type 1 receptor (AT1R). In the present study, we examined the therapeutic effects of olmesartan, an AT1R-specific blocker, in genetically obese and diabetic KKAy mice, a model of human metabolic disorders with diabetes without any dietary loading [8], and focused our analysis on adipose tissue.

\section{Materials and Methods}

2.1. Animals and Treatment. C57BL/6 mice (male) and KKAy mice (male) were purchased from CLEA Japan, Inc. (Tokyo, Japan) for use as a nondiabetic normal control and a model of metabolic disorders with type 2 diabetes, respectively [911]. These mice were housed in a controlled environment with a $12 \mathrm{~h}$ light-dark cycle and were allowed free access to food and water. They were fed a standard diet $(3.6 \mathrm{kcal} / \mathrm{g}$; 13.3\% energy as fat; Oriental MF, Oriental Yeast, Co., Ltd.). Male KKAy mice at 9 weeks of age were treated with the oral administration of olmesartan ( $3 \mathrm{mg} / \mathrm{kg}$ per day) in drinking water for 4 weeks, and body weight and food intake were measured. The KKAy mice treated with vehicle were previously described [12]. On the other hand, C57BL/6 control mice were treated with vehicle during the study period. Mice were sacrificed under anesthesia and the tissues were collected at the end of the experimental period. The protocol was reviewed and approved by the Animal Studies Committee of Yokohama City University and all experiments were performed in accordance with the National Institutes of Health guidelines for the use of experimental animals.

2.2. Blood Pressure Measurement by Tail-Cuff Method. Systolic blood pressure was measured noninvasively by the tail-cuff method (BP-monitor MK-2000; Muromachi Kikai Co.). The MK-2000 BP-monitor allowed determination of the blood pressure without any preheating of the animals, thus avoiding this very stressful condition [13-15]. At least eight readings were taken for each measurement.

2.3. Preparation of Tissue Sections and Histological Analysis. The epididymal white adipose tissue was isolated and fixed with $10 \%$ paraformaldehyde overnight and embedded in paraffin. Tissue sections were stained with hematoxylin and eosin for cell size determination. The adipocyte diameter and area were quantified using Image-Pro Plus software.

2.4. Tissue RNA Isolation and Real-Time Quantitative Reverse Transcript-PCR ( $q R T-P C R)$ Analysis. Total RNA was extracted from epididymal adipose tissue with ISOGEN (Nippon Gene), and cDNA was synthesized using the SuperScript III First-Strand System (Invitrogen). Real-time qRT-PCR was performed with an ABI PRISM 7000 Sequence Detection System by incubating the reverse transcription product with TaqMan PCR Master Mix and a designed TaqMan probe (Applied Biosystems), essentially as described previously [15-18]. The mRNA levels were normalized to those of the $18 \mathrm{~S}$ rRNA control.

2.5. Statistical Analysis. All data are shown as mean \pm SEM. Differences were analyzed by ANOVA followed by the Newman-Keuls multiple-comparison test. A $P$ value of $<0.05$ was considered statistically significant.

\section{Results}

3.1. Effects of Olmesartan on Blood Pressure, Body Weight, and Food Intake. The systolic blood pressure, heart rate, and body weight at baseline and after the study period in the control C57BL/6, vehicle-treated KKAy, and olmesartantreated KKAy mice are shown in Figure 1. At baseline there were no significant differences in systolic blood pressure or heart rate between the groups before the treatment. Body weight at baseline was significantly greater in the KKAy mice of either treatment group than in the control C57BL/6 mice $(P<0.01$ versus $\mathrm{C} 57 \mathrm{BL} / 6)$. With respect to the effects of treatment with olmesartan at a dose of $3 \mathrm{mg} / \mathrm{kg}$ per day for 4 weeks on heart rate and body weight, heart rate was not affected in the KKAy mice by the treatment with olmesartan for 4 weeks compared with baseline (baseline versus 4 weeks, $720 \pm 11$ versus $729 \pm 8$ bpm, NS), and there was a significant increase in body weight after the olmesartan treatment (baseline versus 4 weeks, $41.2 \pm 0.4$ versus $47.5 \pm$ $0.8 \mathrm{~g}, P<0.01)$. After 4 weeks, heart rate and body weight did not differ significantly between the vehicle-treated and olmesartan-treated KKAy mice (Figure 1).

On the other hand, systolic blood pressure in the KKAy mice was significantly decreased by the olmesartan treatment for 4 weeks compared with baseline (baseline versus 4 weeks; $108 \pm 3$ versus $95 \pm 3 \mathrm{mmHg}, P<0.01$ ), and systolic blood pressure was significantly lower in the KKAy mice treated with olmesartan than those treated with vehicle $(P<$ 0.01 versus vehicle) (Figure 1 ). Furthermore, although the daily food intake after 4 weeks was significantly greater in the KKAy mice of either treatment group than the control C57BL/6 mice $(P<0.01$ versus C57BL/6), the daily food intake was similar in the vehicle-treated and olmesartantreated KKAy mice groups (Figure 2).

\subsection{Effects of Olmesartan on Adipocyte Hypertrophy in KKAy} Mice. We examined whether there was any phenotypic alteration in the adipose tissue of the KKAy mice treated with olmesartan. Although the KKAy mice treated with vehicle had significantly larger adipocytes than the control C57BL/6 mice $(P<0.01$ versus C57BL/6) (Figures 3(a), 3(b), and 3(c)), adipocyte hypertrophy was significantly inhibited in the KKAy mice treated with olmesartan for 4 weeks (Figure 3(d); vehicle-treated KKAy mice versus olmesartan-treated KKAy mice; diameter: $113.7 \pm 3.7$ versus $91.2 \pm 3.1 \mu \mathrm{m}, P<0.01$; Figure 3(e), area: $11131 \pm 765$ versus $\left.7264 \pm 415 \mu \mathrm{m}^{2}, P<0.01\right)$.

3.3. Effects of Olmesartan on Adipokine and Adipose Tissue RAS Gene Expression. As shown in Figure 4, the KKAy mice treated with vehicle exhibited significantly suppressed 


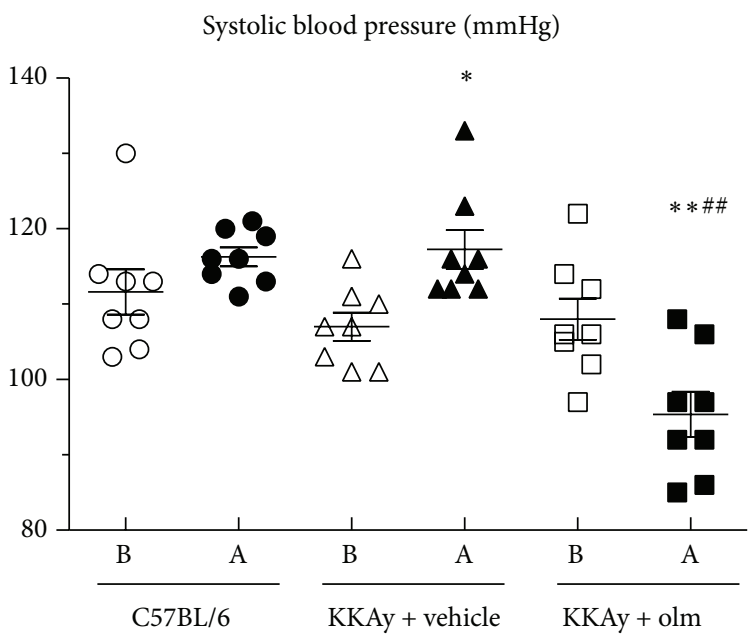

(a)

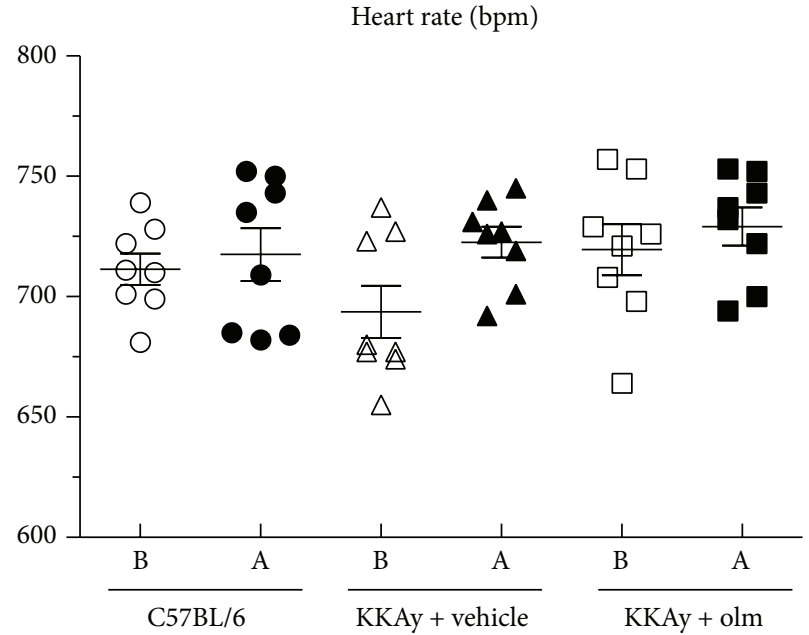

(b)

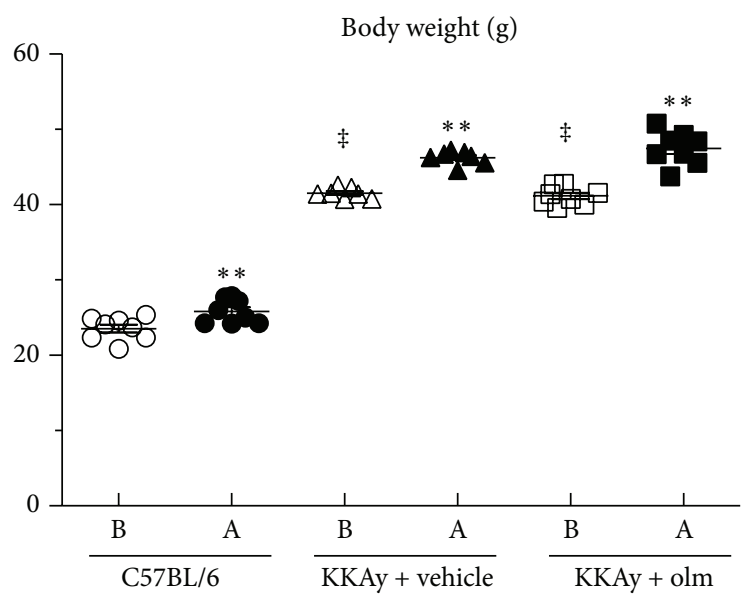

(c)

FIGURE 1: Effects of olmesartan (olm) on systolic blood pressure (a), heart rate (b), and body weight (c) in KKAy mice. Individual values are shown in the graphs and the values are also shown as the mean \pm SEM $(n=8)$. B, before treatment; A, after treatment. ${ }^{*} P<0.05,{ }^{* *} P<0.01$ versus before treatment; ${ }^{\# \#} P<0.01$ versus KKAy + vehicle; ${ }^{\ddagger} P<0.01$ versus C57BL/6 (ANOVA).

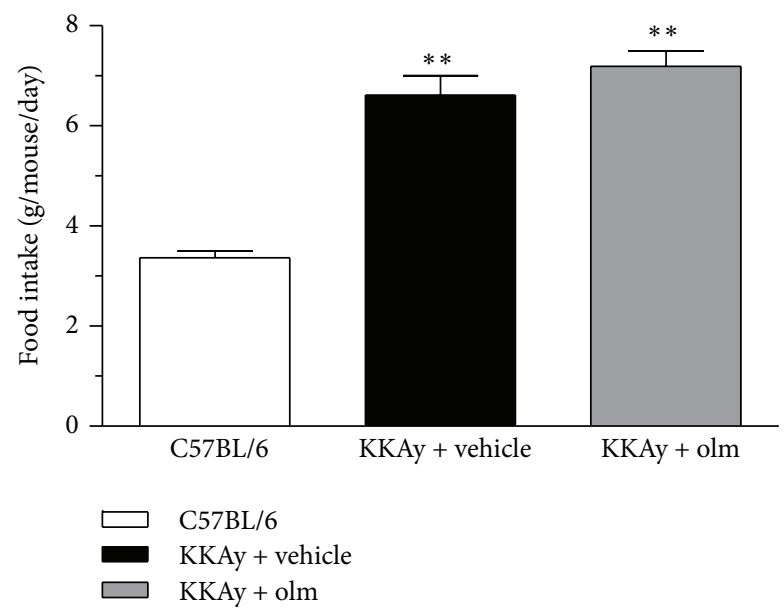

Figure 2: Effects of olmesartan (olm) on daily food intake in KKAy mice. The values are the mean $\pm \operatorname{SEM}(n=8)$. ${ }^{* *} P<0.01$ versus C57BL/6 (ANOVA). 


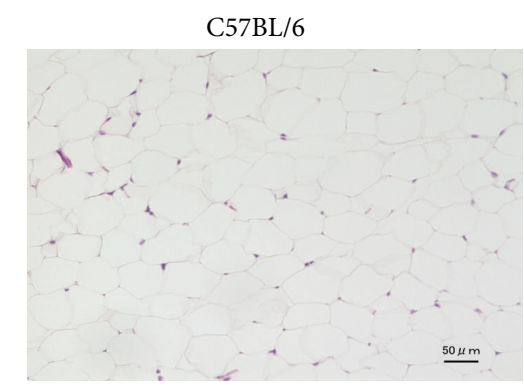

(a)

Adipocyte diameter $(\mu \mathrm{m})$

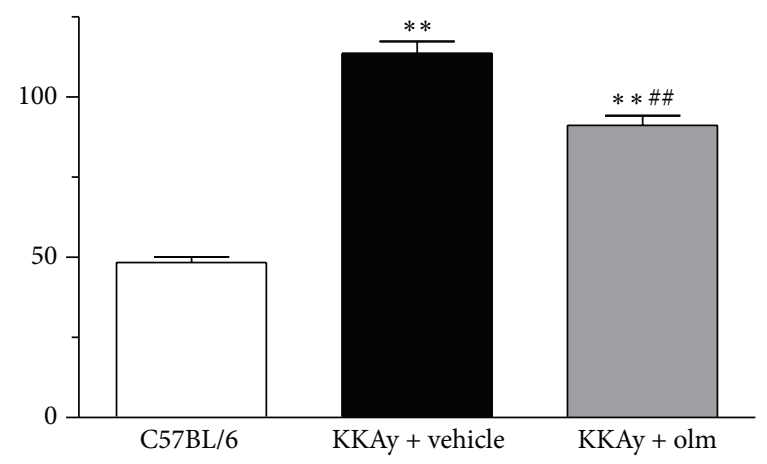

(d)

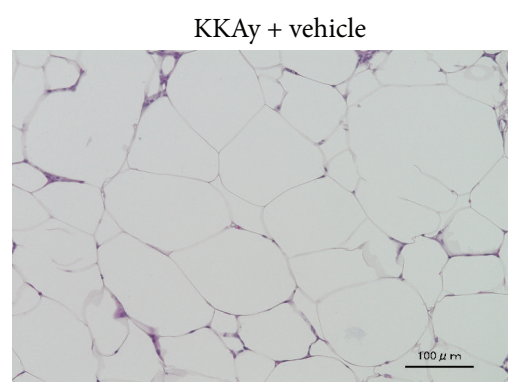

(b)

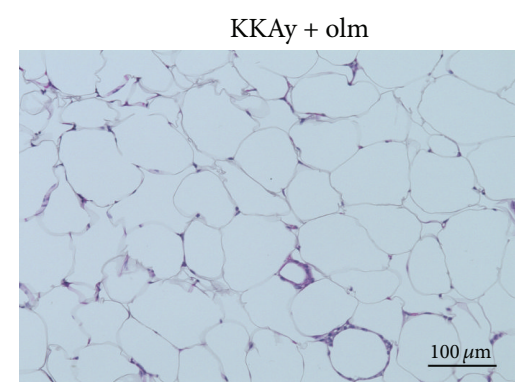

(c)

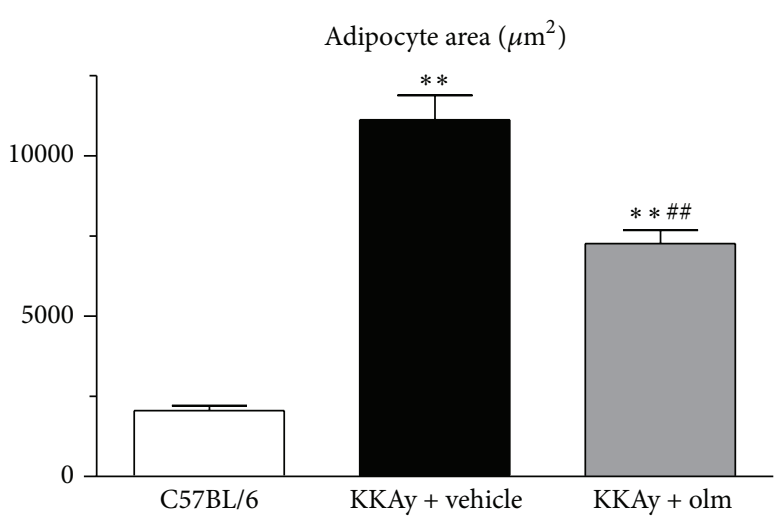

(e)

FIGURE 3: Effects of olmesartan (olm) on adipocyte hypertrophy in KKAy mice. Upper panel: histological analysis of epididymal adipose tissue sections ((a) C57BL/6; (b) KKAy + vehicle; (c) KKAy + olmesartan) stained with hematoxylin and eosin in each experimental group. Original magnification: $\times 200$. Lower panel: adipocyte diameter $(\mathrm{d})$ and area (e). The values are the mean $\pm \operatorname{SEM}(n=8)$. ${ }^{* *} P<0.01$ versus C57BL/6; ${ }^{\# \#} P<0.01$ versus KKAy + vehicle (ANOVA). Olm indicates olmesartan.

Adiponectin

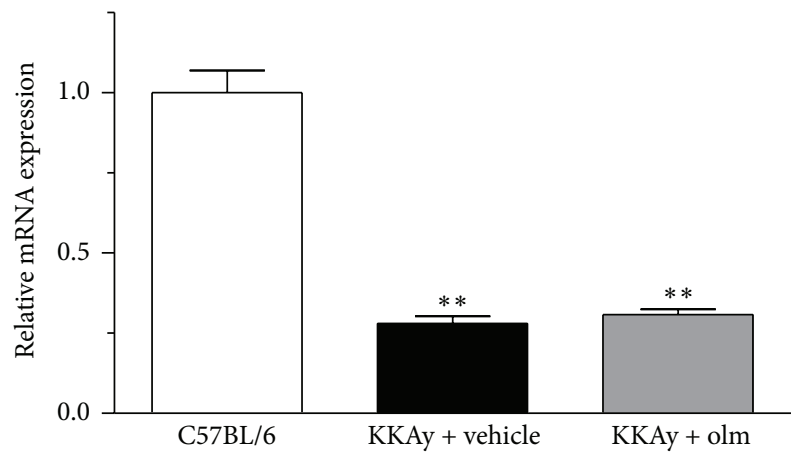

(a)
$\operatorname{PPAR} \gamma$

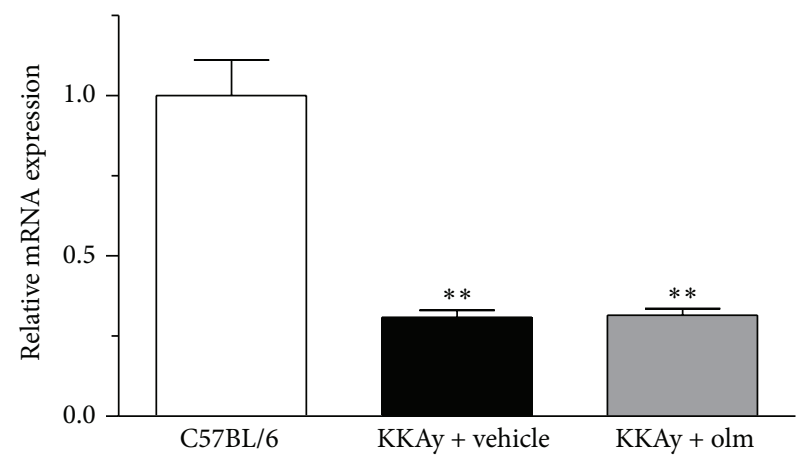

(b)

FIGURE 4: Effects of olmesartan (olm) on the adipose tissue mRNA expression of adiponectin (a) and PPAR $\gamma$ (b) in KKAy mice. The values are the mean $\pm \operatorname{SEM}(n=8) .{ }^{* *} P<0.01$ versus C57BL/6 (ANOVA). Olm indicates olmesartan.

adipose tissue expression of adiponectin, an important adipokine, as well as peroxisome proliferator-activated receptor $\gamma(\operatorname{PPAR} \gamma)$, compared with the control C57BL/6 mice (Figures 4(a) and 4(b)). With respect to a possible effect of olmesartan on adiponectin and $\operatorname{PPAR} \gamma$, the treatment with olmesartan did not affect adiponectin or PPAR $\gamma$ mRNA expression in the adipose tissue of KKAy mice (Figures 4(a) and 4(b)). We also examined the possible influence of olmesartan on adipose tissue expression of the RAS component genes (angiotensinogen, ATRAP, and AT1R) in KKAy mice. While the KKAy mice treated with vehicle exhibited a significantly lower expression of adipose angiotensinogen and ATRAP mRNA than the control C57BL/ 6 mice $(P<0.01$ versus $\mathrm{C} 57 \mathrm{BL} / 6)$, adipose AT1R mRNA expression was not altered in vehicle-treated KKAy mice (Figures 5(a), 5(b), and 5(c)). In addition, treatment with olmesartan did not affect 


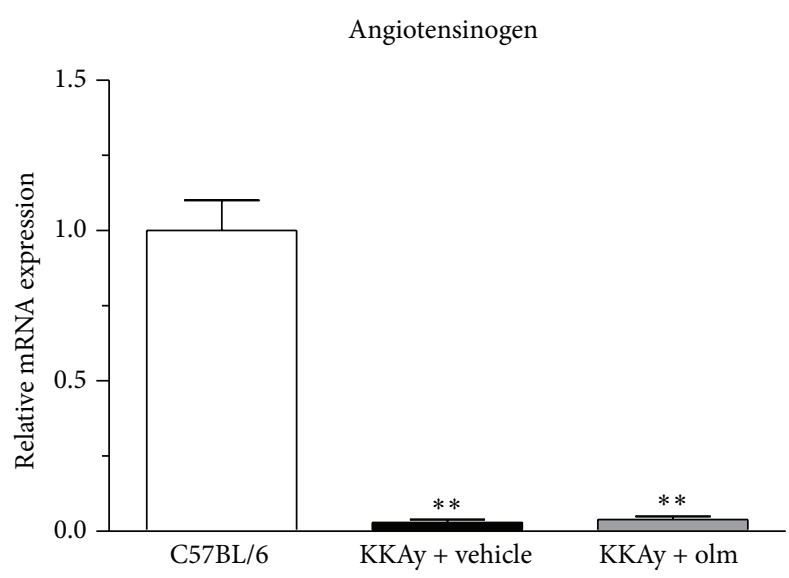

(a)

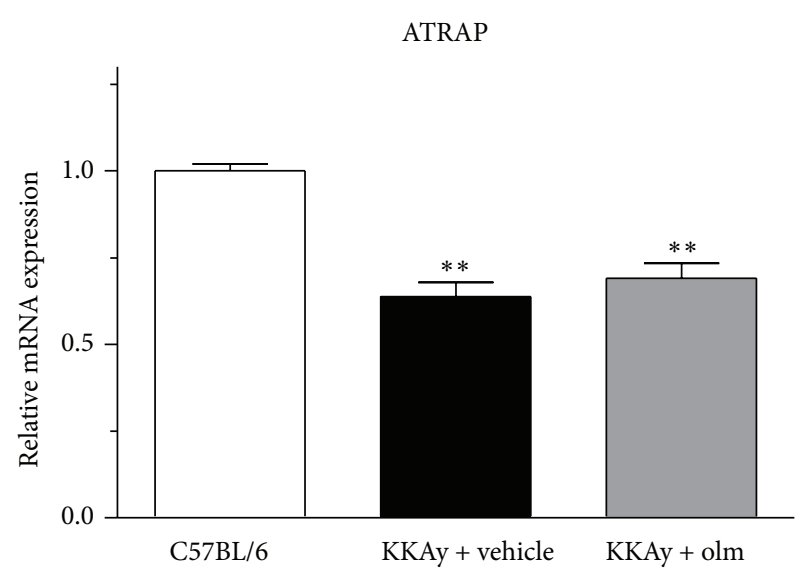

(b)

ATla receptor

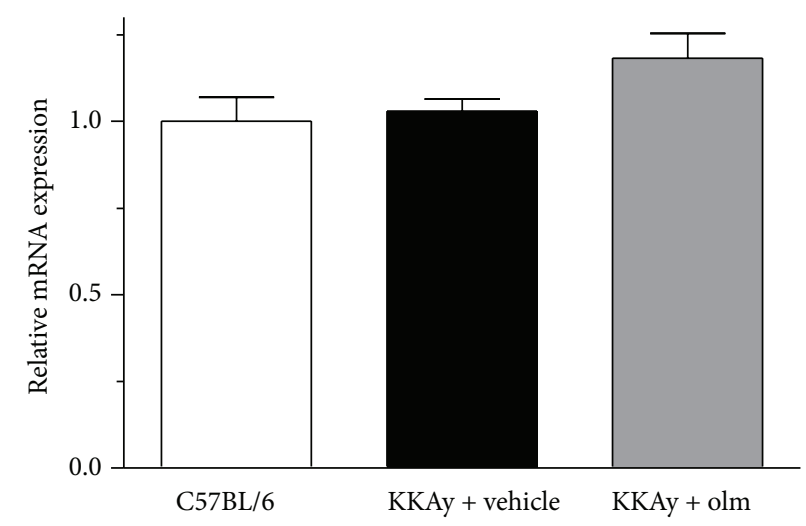

(c)

Figure 5: Effects of olmesartan (olm) on the adipose tissue mRNA expression of angiotensinogen (a), ATRAP (b), and AT1R (c) (AT1a receptor) in KKAy mice. The values are the mean $\pm \operatorname{SEM}(n=8) .{ }^{* *} P<0.01$ versus C57BL/6 (ANOVA). Olm indicates olmesartan.

the expression of angiotensinogen, AT1R, or ATRAP mRNA in the adipose tissue of KKAy mice (Figures 5(a), 5(b), and $5(\mathrm{c})$ ).

3.4. Effects of Olmesartan on Adipose Tissue Inflammatory Cytokines. Regarding the expression of tissue inflammatory cytokines (MCP-1, TNF- $\alpha$, IL-6, and PAI-1) in adipose tissue (Figure 6), while the PAI-1 mRNA expression was not altered in the vehicle-treated KKAy mice (Figure 6(d)), the mRNA levels of MCP-1, TNF- $\alpha$, and IL- 6 were all significantly upregulated in the KKAy mice treated with vehicle compared with the control C57BL/ 6 mice (MCP-1 and TNF- $\alpha, P<0.01$; IL-6, $P<0.05$ versus C57BL/6) (Figures 6(a), 6(b), and $6(c))$. With respect to a possible effect of olmesartan on these inflammatory cytokine genes in the adipose tissue, the KKAy mice treated with olmesartan exhibited a blunted increase in adipose IL-6 mRNA expression (Figure 6(c)), in spite of the fact that there are no effects on the adipose MCP-1, TNF- $\alpha$, and PAI-1 mRNA expression (Figures 6(a), 6(b), and 6(d)).

3.5. Effects of Olmesartan on Adipose Tissue Oxidative Stress. We finally examined the possible effects of olmesartan on the expression of the NADPH oxidase components (p22phox, gp91phox, p47phox, and p40phox) in the epididymal adipose tissue of KKAy mice. As shown in Figure 7, although the vehicle-treated KKAy mice exhibited a significantly elevated expression of these NADPH oxidase component mRNA levels in the adipose tissue compared with the control C57BL/6 mice (p22phox, gp91phox, p47phox, and p40phox, $P<0.01$ versus C57BL/6), treatment with olmesartan for 4 weeks significantly suppressed the enhanced adipose tissue expression of p22phox, gp91phox, and p47phox mRNA in KKAy mice without affecting adipose p40phox mRNA expression mice (p22phox and gp91phox, $P<0.01$; 4 47phox, $P<0.05$ versus vehicle) (Figures 7(a), 7(b), 7(c), and 7(d)).

\section{Discussion}

Increasing evidence has indicated that adipose tissue is profoundly involved in the physiological and pathophysiological regulation of circulatory and endocrine systems in vivo via modulatory effects on the local production of inflammatory cytokines, adipokines, and vasoactive factors. In addition, it has been demonstrated that the genes of RAS components such as angiotensinogen and AT1R were substantially expressed in adipose tissue [6]. The local RAS 


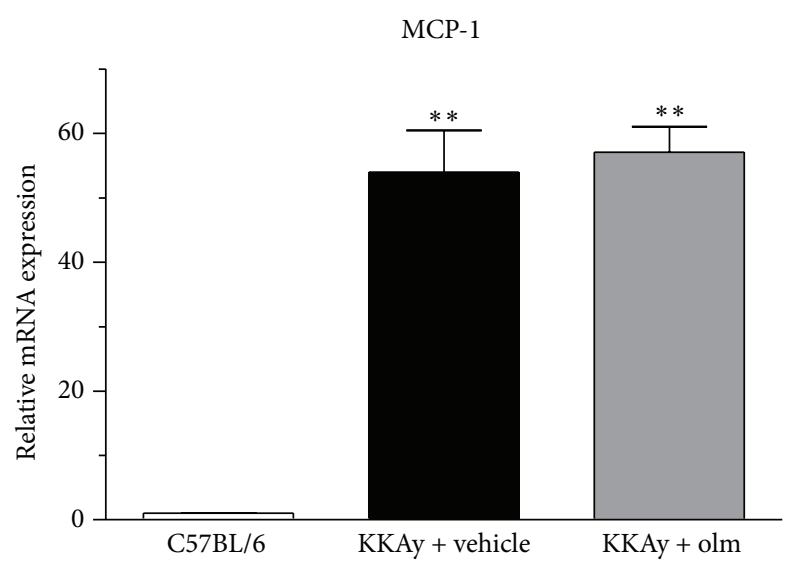

(a)

IL-6

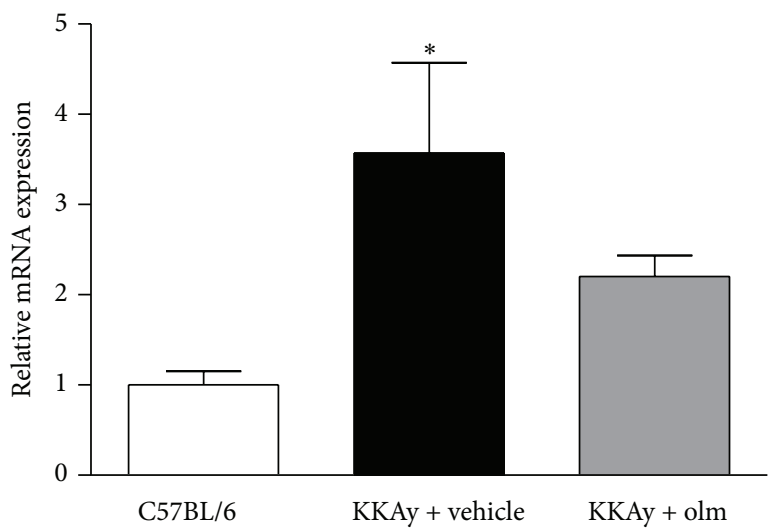

(c)

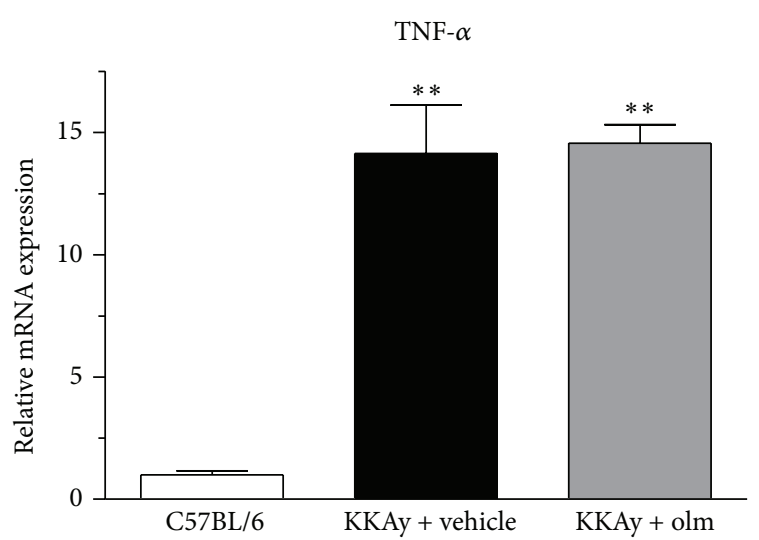

(b)

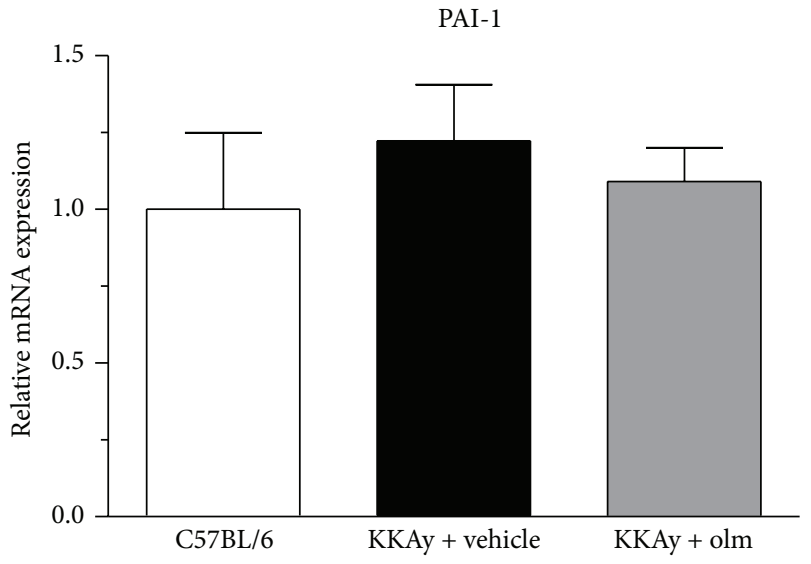

(d)

FIGURE 6: Effects of olmesartan (olm) on the adipose tissue mRNA expression of proinflammatory cytokines ((a) MCP-1; (b) TNF- $\alpha$; (c) IL-6; and (d) PAI-1) in KKAy mice. The values are the mean $\pm \operatorname{SEM}(n=8) .{ }^{*} P<0.05,{ }^{* *} P<0.01$ versus C57BL/6 (ANOVA). Olm indicates olmesartan.

in adipose tissue is suggested to be critically involved in the modulation of the physiological function of adipocytes, and furthermore, the pathological activation of adipose tissue RAS reportedly plays a crucial role in the pathophysiology of metabolic disorders via the dysregulated production of oxidative stress, inflammatory cytokines, and adipokines in adipose tissue $[7,19]$. Thus, it is certainly important to identify any beneficial effects of interventions on adipose tissue in order to develop a more efficient therapeutic strategy to treat metabolic disorders with obesity.

In the present study, 4-week olmesartan treatment significantly decreased blood pressure in KKAy mice, a human model of metabolic disorders, without any significant effects on dietary food intake or body weight gain. Thus, the hypotensive effect of olmesartan was exerted without any inhibitory effect on body weight gain in KKAy mice. However, from the point of view of possible pleiotropic effects of olmesartan on adipose tissue function, there are three interesting findings possibly related to adipose tissue in KKAy mice: (1) an inhibitory effect on adipocyte hypertrophy, (2) a suppressive effect on IL-6 gene expression, and (3) an ameliorating effect on oxidative stress.

Previous studies showed that the persistent low-grade activation of chronic inflammatory responses in adipose tissue plays an important role in the development of metabolic disorders with visceral obesity [20-26] and that chronic adipose tissue inflammation is provoked via the stimulated secretion of proinflammatory cytokines and factors derived from adipocytes [4, 27]. Although adiponectin is a wellestablished adipocyte-secreted endocrine factor involved in the pathophysiology of metabolic disorders and provides a functional link between adipose tissue and the immune system $[5,28]$, the circulating adiponectin level is reportedly decreased in metabolic disorders with visceral obesity [29]. In addition, PPAR $\gamma$ is reported to improve adipocytokine dysregulation in adipose tissue, including adiponectin, in metabolic disorders [30]. In the present study, while the treatment of KKAy mice with olmesartan did not affect the adipose expression of adiponectin or PPAR $\gamma$, olmesartan inhibited the adipose tissue gene expression of IL-6, which 


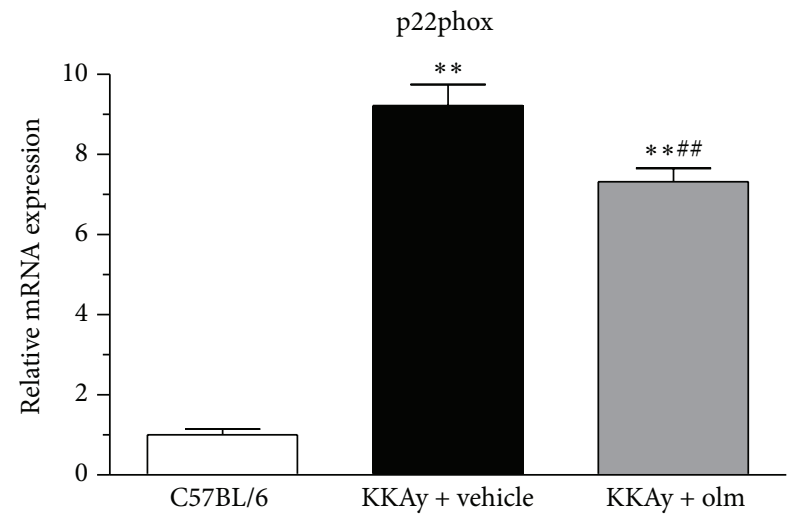

(a)

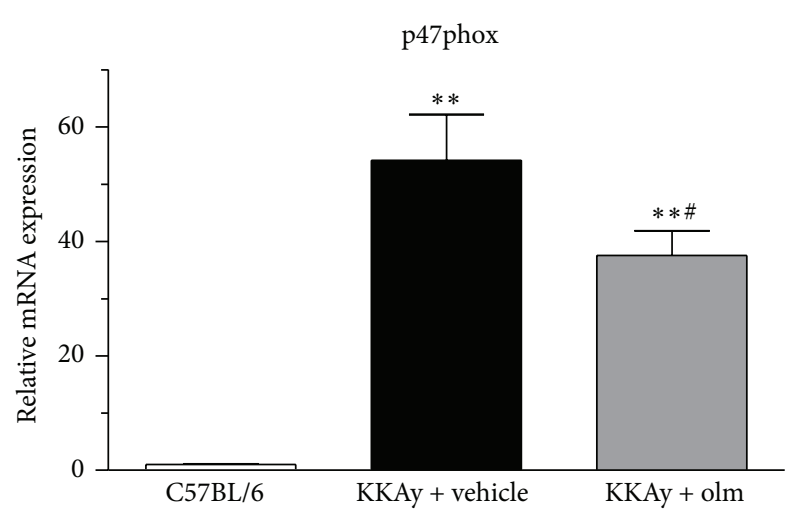

(c)

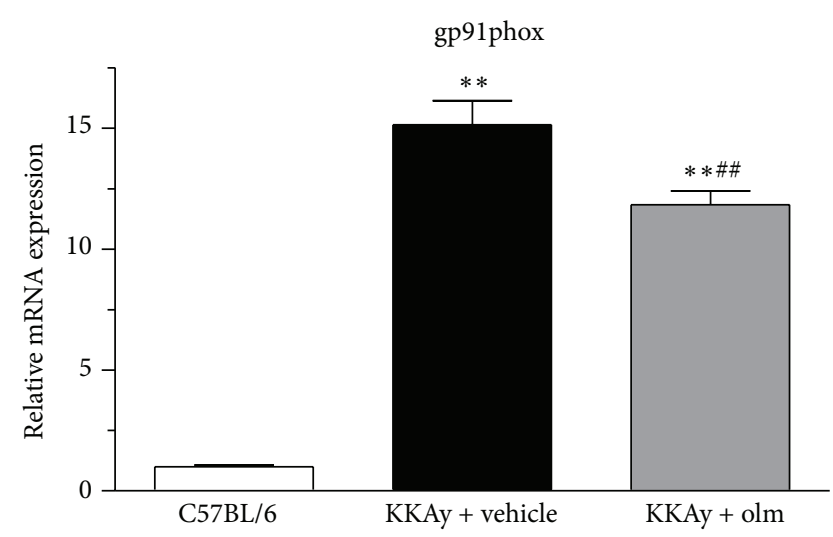

(b)

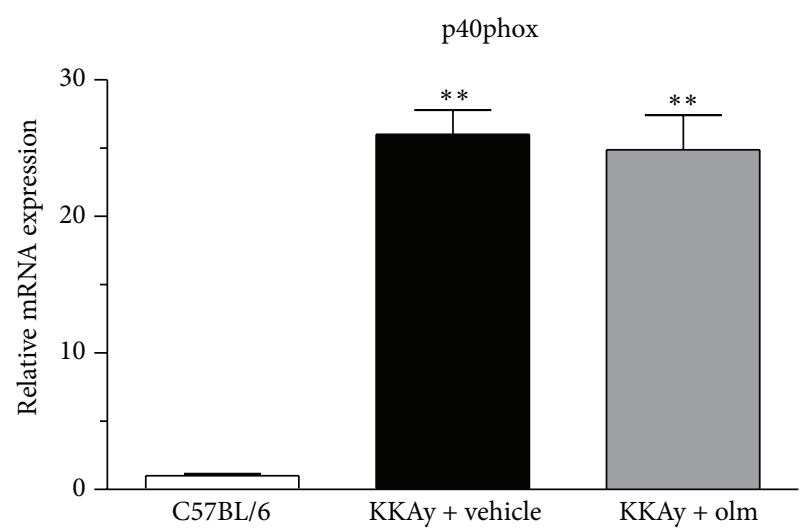

(d)

FIGURE 7: Effects of olmesartan (olm) on the adipose tissue mRNA expression of NADPH oxidase components ((a) p22phox; (b) gp91phox; (c) p47phox; and (d) p40phox) in KKAy mice. The values are the mean $\pm \operatorname{SEM}(n=8) .{ }^{* *} P<0.01$ versus C57BL/6; ${ }^{\#} P<0.05$, ${ }^{\# \#} P<0.01$ versus KKAy + vehicle (ANOVA). Olm indicates olmesartan.

is also one of the key players in the inflammatory process in adipose tissue in metabolic disorders [22, 31].

Accumulated adipose tissue-induced dysregulated production of adipocytokines, including proinflammatory cytokines such as IL-6, is reported to activate NADPH oxidase components [32-35]. Adipose NADPH oxidase-derived reactive oxygen species (ROS) function as important intracellular second messengers to activate many downstream signaling molecules that modulate endothelial function, pathological growth and migration of vascular cells, expression of proinflammatory mediators and modification of extracellular matrix [36-39]. All of these processes play important roles in the development of insulin resistance and cardiovascular disease in metabolic disorders with visceral obesity. In the present study, olmesartan exerted a suppressive effect on adipocyte hypertrophy concomitant with an inhibitory effect on the IL-6-oxidative stress axis without any body weight reducing effect in KKAy mice, a human model of metabolic disorders.

We previously identified ATRAP as a novel molecule interacting with AT1R and showed that ATRAP suppressed the Ang II-induced pathological responses of cardiovascular cells and tissues by promoting AT1R internalization $[17,18,40-42]$. Thus, a tissue-specific regulatory balancing of ATRAP and AT1R expression may be involved in the modulation of AT1R signaling that specifically occurs in each tissue [43-46]. We showed that the upregulation of the cardiac ATRAP/AT1R ratio is one of the therapeutic benefits of olmesartan in inhibiting cardiac hypertrophy in hypertensive rats [47]. In addition, prepubertal transient blockade of AT1R signaling by olmesartan exerted a longterm therapeutic effect on salt-induced hypertension and renal injury in Dahl Iwai salt-sensitive rats, partly through a sustained enhancement of renal ATRAP expression [48].

Furthermore, a recent study employing mice with the gene-targeted systemic deletion of ATRAP has shown that the development of systemic insulin resistance related to ATRAP deficiency is attributable to the exaggerated adipose tissue inflammation that occurs via the secretion of proinflammatory cytokines and factors derived from enlarged adipocytes, thereby suggesting ATRAP to be a novel molecular target in metabolic disorders in visceral obesity [19]. However, in the present study, the treatment with olmesartan exerted no evident influence on adipose tissue ATRAP gene expression in KKAy mice. Therefore, further studies are necessary to examine whether the adipose ATRAP is involved in the olmesartan-induced beneficial suppressive effect on the IL-6oxidative stress axis in KKAy mice. 
In the present study, the body weight did not differ significantly between the vehicle-treated and olmesartantreated KKAy mice after 4 weeks. A previous study showed that olmesartan treatment at a dose of $10 \mathrm{mg} / \mathrm{kg}$ per day for 6 weeks exerted an inhibitory effect on body weight gain with a trend of reduction in adiposity without any evident change in food intake in obese Otsuka Long-Evans Tokushima Fatty rats [49]. In contrast, another study reported that treatment with regular chow containing $0.0015 \%$ olmesartan for 2 weeks resulted in a significant reduction in blood pressure level without any effect on food intake or body weight gain in KKAy mice [50]. These results suggest that the inhibitory effects of olmesartan on body weight and adipose tissue mass may depend on the diabetic animal model used and the condition of olmesartan treatment (dose and duration). Furthermore, as a limitation of the present study, although olmesartan significantly suppressed the enhanced adipose tissue mRNA expression of NADPH oxidase, this does not constitute direct evidence of an effect. Further studies on the protein expression of NADPH oxidase and adipose tissue production of oxidative stress are needed to obtain a definitive result.

\section{Conclusions}

In summary, the results of the present study in a mouse model of human metabolic disorders showed a therapeutic effect of olmesartan on adipose tissue in addition to its blood pressure lowering effect. The results suggest that the olmesartanmediated inhibitory effect on adipocyte hypertrophy in KKAy mice is associated with a beneficial suppression of the IL6-oxidative stress axis in adipose tissue. Further studies are needed to demonstrate this beneficial effect of olmesartan on adipose tissue oxidative stress and to clarify whether there is a functional role of adipose ATRAP in the pathophysiology of metabolic disorders.

\section{Disclosure}

Kouichi Tamura has received honoraria, consulting fees, or funds from Novartis, Takeda, Daiichi-Sankyo, DainipponSumitomo, Kyowa-Hakko Kirin, Chugai, Shionogi, Boehringer Ingelheim, Astellas, Mochida, Pfizer, Mitsubishi Tanabe, and Sanofi.

\section{Conflict of Interests}

The authors declare that there is no conflict of interests regarding the publication of this paper.

\section{Acknowledgments}

This work was supported by a Health and Labor Sciences Research Grant and by Grants from the Japanese Ministry of Education, Science, Sports and Culture, the Salt Science Research Foundation (no. 1428), the Yokohama Foundation for Advancement of Medical Science, the Kidney Foundation,
Japan (JKFB13-17), and the Novartis Foundation for Gerontological Research (2012). Pacific Edit reviewed the paper prior to submission.

\section{References}

[1] J. P. Reis, C. M. Loria, C. E. Lewis et al., "Association between duration of overall and abdominal obesity beginning in young adulthood and coronary artery calcification in middle age," Journal of the American Medical Association, vol. 310, no. 3, pp. 280-288, 2013.

[2] E. Maury and S. M. Brichard, "Adipokine dysregulation, adipose tissue inflammation and metabolic syndrome," Molecular and Cellular Endocrinology, vol. 314, no. 1, pp. 1-16, 2010.

[3] G. R. Hajer, T. W. van Haeften, and F. L. J. Visseren, "Adipose tissue dysfunction in obesity, diabetes, and vascular diseases," European Heart Journal, vol. 29, no. 24, pp. 2959-2971, 2008.

[4] N. Ouchi, J. L. Parker, J. J. Lugus, and K. Walsh, "Adipokines in inflammation and metabolic disease," Nature Reviews Immunology, vol. 11, no. 2, pp. 85-97, 2011.

[5] H. S. Mattu and H. S. Randeva, "Role of adipokines in cardiovascular disease," Journal of Endocrinology, vol. 216, no. 1, pp. T17-T36, 2013.

[6] L. Yvan-Charvet and A. Quignard-Boulangé, "Role of adipose tissue renin-angiotensin system in metabolic and inflammatory diseases associated with obesity," Kidney International, vol. 79, no. 2, pp. 162-168, 2011.

[7] F. Jing, M. Mogi, and M. Horiuchi, "Role of renin-angiotensinaldosterone system in adipose tissue dysfunction," Molecular and Cellular Endocrinology, vol. 378, no. 1-2, pp. 23-28, 2013.

[8] J.-I. Suto, S. Matsuura, K. Imamura, H. Yamanaka, and K. Sekikawa, "Genetic analysis of non-insulin-dependent diabetes mellitus in KK and KK-A(y) mice," European Journal of Endocrinology, vol. 139, no. 6, pp. 654-661, 1998.

[9] M. Iwai, H. Kanno, Y. Tomono et al., "Direct renin inhibition improved insulin resistance and adipose tissue dysfunction in type 2 diabetic KK-Ay mice," Journal of Hypertension, vol. 28, no. 7, pp. 1471-1481, 2010.

[10] F. Jing, M. Mogi, L.-J. Min et al., "Effect of angiotensin II type 2 receptor-interacting protein on adipose tissue function via modulation of macrophage polarization," PLOS ONE, vol. 8, no. 4, Article ID e60067, 2013.

[11] B. Lei, D. Nakano, Y.-Y. Fan et al., "Add-on aliskiren elicits stronger renoprotection than high-dose valsartan in type 2 diabetic KKAy mice that do not respond to low-dose valsartan," Journal of Pharmacological Sciences, vol. 119, no. 2, pp. 131-138, 2012.

[12] A. Maeda, K. Tamura, H. Wakui et al., "Effects of Ang II receptor blocker irbesartan on adipose tissue function in mice with metabolic disorders," International Journal of Medical Sciences, vol. 11, no. 6, pp. 646-651, 2014.

[13] T. Iwamoto, S. Kita, J. Zhang et al., "Salt-sensitive hypertension is triggered by $\mathrm{Ca}^{2+}$ entry via $\mathrm{Na}^{+} / \mathrm{Ca}^{2+}$ exchanger type- 1 in vascular smooth muscle," Nature Medicine, vol. 10, no. 11, pp. 1193-1199, 2004.

[14] Y. Tsurumi, K. Tamura, Y. Tanaka et al., "Interacting molecule of AT1 receptor, ATRAP, is colocalized with AT1 receptor in the mouse renal tubules," Kidney International, vol. 69, no. 3, pp. 488-494, 2006.

[15] H. Wakui, K. Tamura, M. Matsuda et al., "Intrarenal suppression of angiotensin II type 1 receptor binding molecule in 
angiotensin II-infused mice," American Journal of Physiology: Renal Physiology, vol. 299, no. 5, pp. F991-F1003, 2010.

[16] M. Matsuda, K. Tamura, H. Wakui et al., "Upstream stimulatory factors 1 and 2 mediate the transcription of angiotensin II binding and inhibitory protein," Journal of Biological Chemistry, vol. 288, no. 26, pp. 19238-19249, 2013.

[17] H. Wakui, K. Tamura, Y. Tanaka et al., "Cardiac-specific activation of angiotensin II type 1 receptor-associated protein completely suppresses cardiac hypertrophy in chronic angiotensin II-infused mice," Hypertension, vol. 55, no. 5, pp. 1157-1164, 2010.

[18] H. Wakui, K. Tamura, S.-I. Masuda et al., "Enhanced angiotensin receptor-associated protein in renal tubule suppresses angiotensin-dependent hypertension," Hypertension, vol. 61, no. 6, pp. 1203-1210, 2013.

[19] A. Maeda, K. Tamura, H. Wakui et al., "Angiotensin receptorbinding protein ATRAP/Agtrap inhibits metabolic dysfunction with visceral obesity," Journal of the American Heart Association, vol. 2, no. 4, Article ID e000312, 2013.

[20] C. M. Apovian, S. Bigornia, M. Mott et al., "Adipose macrophage infiltration is associated with insulin resistance and vascular endothelial dysfunction in obese subjects," Arteriosclerosis, Thrombosis, and Vascular Biology, vol. 28, no. 9, pp. 1654-1659, 2008.

[21] J. G. Neels and J. M. Olefsky, "Inflamed fat: what starts the fire?" Journal of Clinical Investigation, vol. 116, no. 1, pp. 33-35, 2006.

[22] S. Schenk, M. Saberi, and J. M. Olefsky, "Insulin sensitivity: modulation by nutrients and inflammation," Journal of Clinical Investigation, vol. 118, no. 9, pp. 2992-3002, 2008.

[23] O. Osborn and J. M. Olefsky, "The cellular and signaling networks linking the immune system and metabolism in disease," Nature Medicine, vol. 18, no. 3, pp. 363-374, 2012.

[24] H. Sell, C. Habich, and J. Eckel, "Adaptive immunity in obesity and insulin resistance," Nature Reviews Endocrinology, vol. 8, no. 12, pp. 709-716, 2012.

[25] M. D. Fullerton, G. R. Steinberg, and J. D. Schertzer, "Immunometabolism of AMPK in insulin resistance and atherosclerosis," Molecular and Cellular Endocrinology, vol. 366, no. 2, pp. 224-234, 2013.

[26] A. W. Ferrante Jr., "Macrophages, fat, and the emergence of immunometabolism," Journal of Clinical Investigation, vol. 123, no. 12, pp. 4992-4993, 2013.

[27] M. Tabata, T. Kadomatsu, S. Fukuhara et al., "Angiopoietin-like protein 2 promotes chronic adipose tissue inflammation and obesity-related systemic insulin resistance," Cell Metabolism, vol. 10, no. 3, pp. 178-188, 2009.

[28] T. Yamauchi, J. Kamon, H. Waki et al., "The fat-derived hormone adiponectin reverses insulin resistance associated with both lipoatrophy and obesity," Nature Medicine, vol. 7, no. 8, pp. 941-946, 2001.

[29] T. Kadowaki and T. Yamauchi, "Adiponectin receptor signaling: a new layer to the current model," Cell Metabolism, vol. 13, no. 2, pp. 123-124, 2011.

[30] N. Maeda, M. Takahashi, T. Funahashi et al., "PPAR $\gamma$ ligands increase expression and plasma concentrations of adiponectin, an adipose-derived protein," Diabetes, vol. 50, no. 9, pp. 20942099, 2001.

[31] M. A. Lazar, "The humoral side of insulin resistance," Nature Medicine, vol. 12, no. 1, pp. 43-44, 2006.

[32] S. Furukawa, T. Fujita, M. Shimabukuro et al., "Increased oxidative stress in obesity and its impact on metabolic syndrome," Journal of Clinical Investigation, vol. 114, no. 12, pp. 1752-1761, 2004.
[33] C. K. Roberts, R. J. Barnard, R. K. Sindhu, M. Jurczak, A. Ehdaie, and N. D. Vaziri, "Oxidative stress and dysregulation of $\mathrm{NAD}(\mathrm{P}) \mathrm{H}$ oxidase and antioxidant enzymes in diet-induced metabolic syndrome," Metabolism: Clinical and Experimental, vol. 55, no. 7, pp. 928-934, 2006.

[34] A. Fernández-Sánchez, E. Madrigal-Santillán, M. Bautista et al., "Inflammation, oxidative stress, and obesity," International Journal of Molecular Sciences, vol. 12, no. 5, pp. 3117-3132, 2011.

[35] W. S. Hahn, J. Kuzmicic, J. S. Burrill et al., "Pro-inflammatory cytokines differentially regulate adipocyte mitochondrial metabolism, oxidative stress and dynamics.", American Journal of Physiology: Endocrinology and Metabolism, vol. 306, no. 9, pp. E1033-E1045, 2014.

[36] G.-X. Zhang, X.-M. Lu, S. Kimura, and A. Nishiyama, "Role of mitochondria in angiotensin II-induced reactive oxygen species and mitogen-activated protein kinase activation," Cardiovascular Research, vol. 76, no. 2, pp. 204-212, 2007.

[37] Y. S. Bae, H. Oh, S. G. Rhee, and Y. D. Yoo, "Regulation of reactive oxygen species generation in cell signaling," Molecules and Cells, vol. 32, no. 6, pp. 491-509, 2011.

[38] B. Lassègue, A. San Martín, and K. K. Griendling, "Biochemistry, physiology, and pathophysiology of NADPH oxidases in the cardiovascular system," Circulation Research, vol. 110, no. 10, pp. 1364-1390, 2012.

[39] A. Schramm, P. Matusik, G. Osmenda, and T. J. Guzik, "Targeting NADPH oxidases in vascular pharmacology," Vascular pharmacology, vol. 56, no. 5-6, pp. 216-231, 2012.

[40] K. Azuma, K. Tamura, A.-I. Shigenaga et al., "Novel regulatory effect of angiotensin II type 1 receptor-interacting molecule on vascular smooth muscle cells," Hypertension, vol. 50, no. 5, pp. 926-932, 2007.

[41] Y. Tanaka, K. Tamura, Y. Koide et al., "The novel angiotensin II type 1 receptor (AT1R)-associated protein ATRAP downregulates AT1R and ameliorates cardiomyocyte hypertrophy," FEBS Letters, vol. 579, no. 7, pp. 1579-1586, 2005.

[42] H. Wakui, T. Dejima, K. Tamura et al., "Activation of angiotensin II type 1 receptor-associated protein exerts an inhibitory effect on vascular hypertrophy and oxidative stress in angiotensin IImediated hypertension," Cardiovascular Research, vol. 100, no. 3, pp. 511-519, 2013.

[43] M. Horiuchi, J. Iwanami, and M. Mogi, "Regulation of angiotensin II receptors beyond the classical pathway," Clinical Science, vol. 123, no. 4, pp. 193-203, 2012.

[44] M. Mogi, M. Iwai, and M. Horiuchi, "Emerging concepts of regulation of angiotensin II receptors: new players and targets for traditional receptors," Arteriosclerosis, Thrombosis, and Vascular Biology, vol. 27, no. 12, pp. 2532-2539, 2007.

[45] K. Tamura, Y. Tanaka, Y. Tsurumi et al., "The role of angiotensin AT1 receptor associated protein in renin-angiotensin system regulation and function," Current Hypertension Reports, vol. 9, no. 2, pp. 121-127, 2007.

[46] K. Tamura, H. Wakui, A. Maeda et al., "The physiology and pathophysiology of a novel angiotensin receptor-binding protein ATRAP/Agtrap," Current Pharmaceutical Design, vol. 19, no. 17, pp. 3043-3048, 2013.

[47] A.-I. Shigenaga, K. Tamura, H. Wakui et al., "Effect of olmesartan on tissue expression balance between angiotensin II receptor and its inhibitory binding molecule," Hypertension, vol. 52, no. 4, pp. 672-678, 2008.

[48] T. Dejima, K. Tamura, H. Wakui et al., "Prepubertal angiotensin blockade exerts long-term therapeutic effect through sustained 
ATRAP activation in salt-sensitive hypertensive rats," Journal of Hypertension, vol. 29, no. 10, pp. 1919-1929, 2011.

[49] R. Rodriguez, J. A. Viscarra, J. N. Minas, D. Nakano, A. Nishiyama, and R. M. Ortiz, "Angiotensin receptor blockade increases pancreatic insulin secretion and decreases glucose intolerance during glucose supplementation in a model of metabolic syndrome," Endocrinology, vol. 153, no. 4, pp. 16841695, 2012.

[50] A. Kurata, H. Nishizawa, S. Kihara et al., "Blockade of angiotensin II type-1 receptor reduces oxidative stress in adipose tissue and ameliorates adipocytokine dysregulation," Kidney International, vol. 70, no. 10, pp. 1717-1724, 2006. 

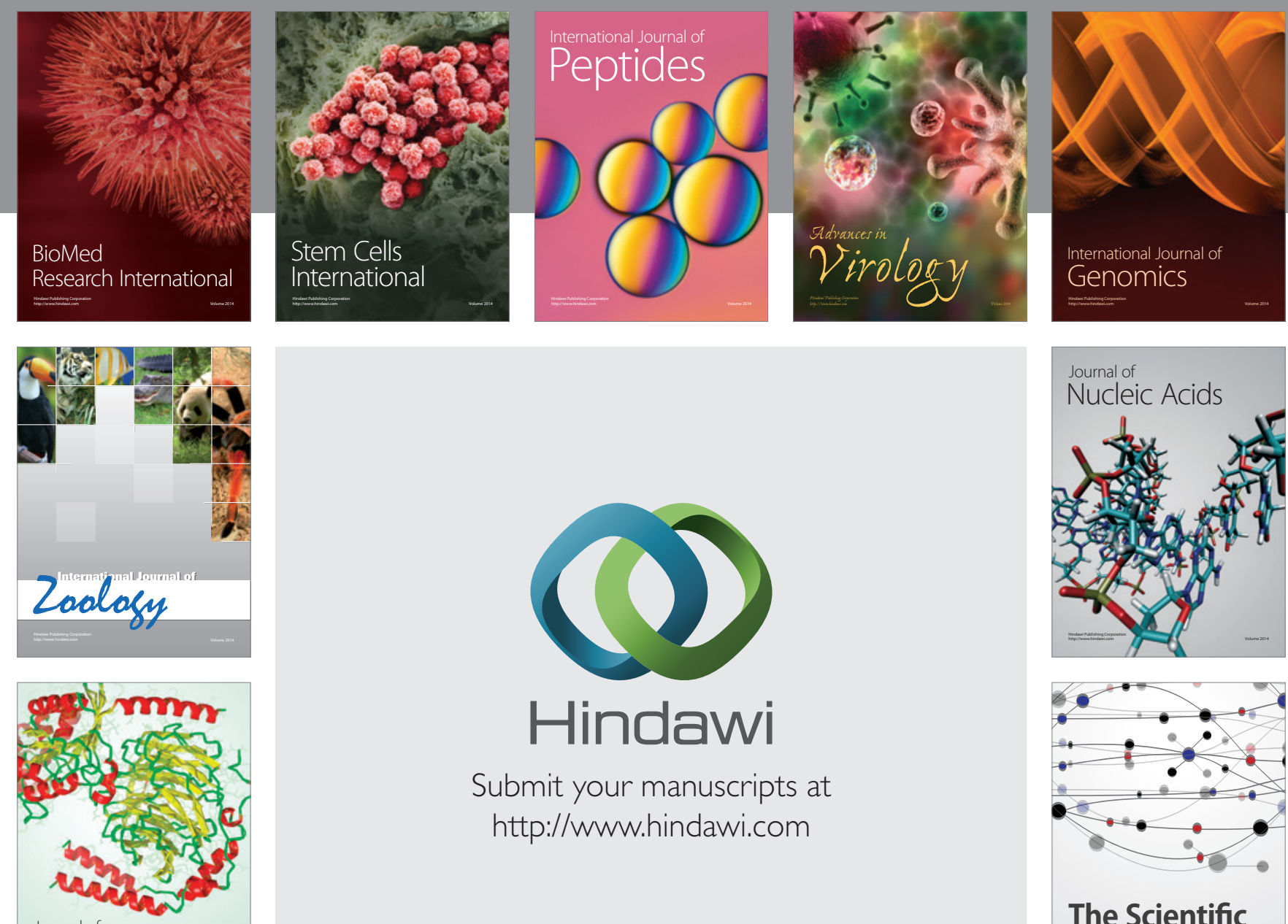

Submit your manuscripts at

http://www.hindawi.com

Journal of
Signal Transduction
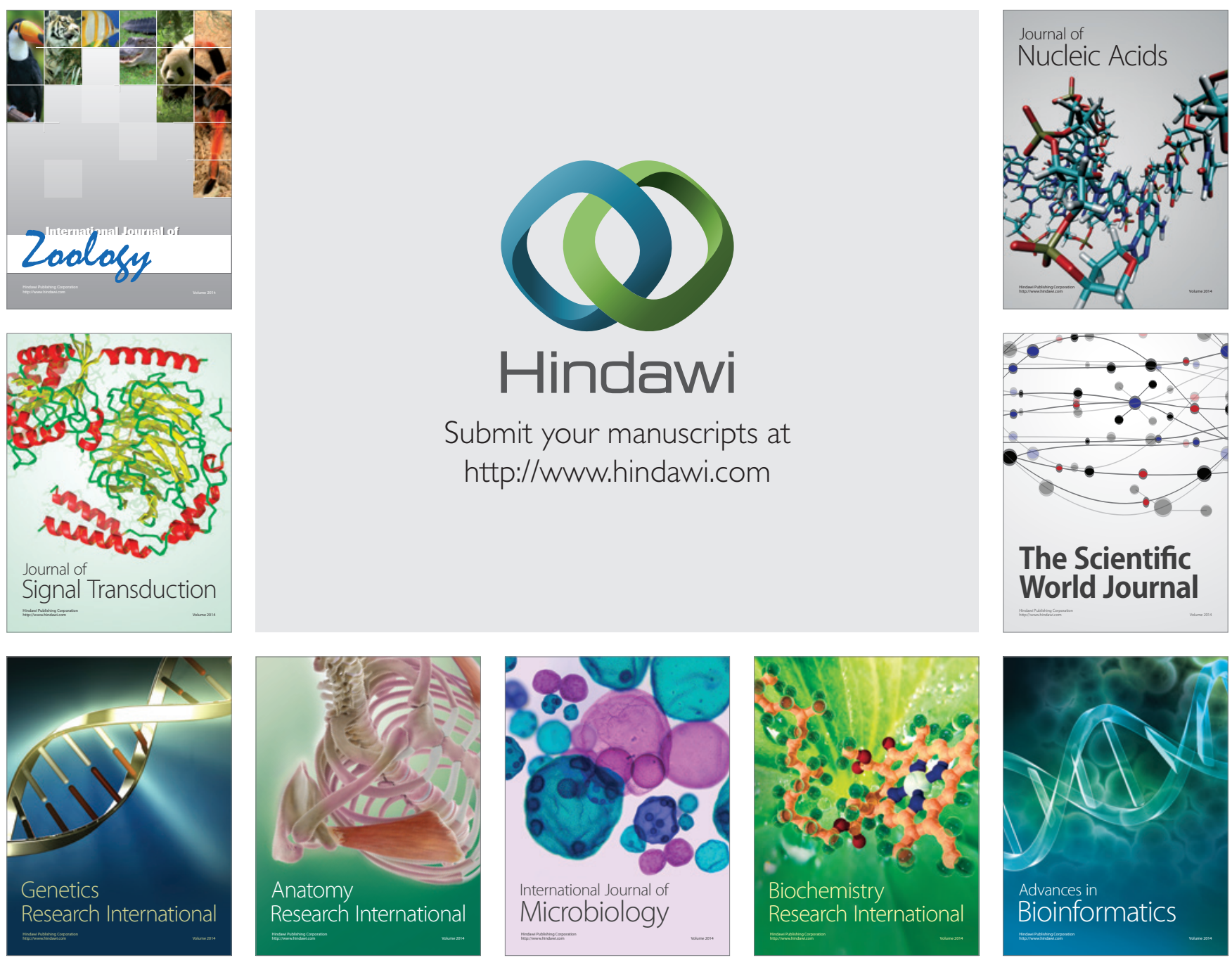

The Scientific World Journal
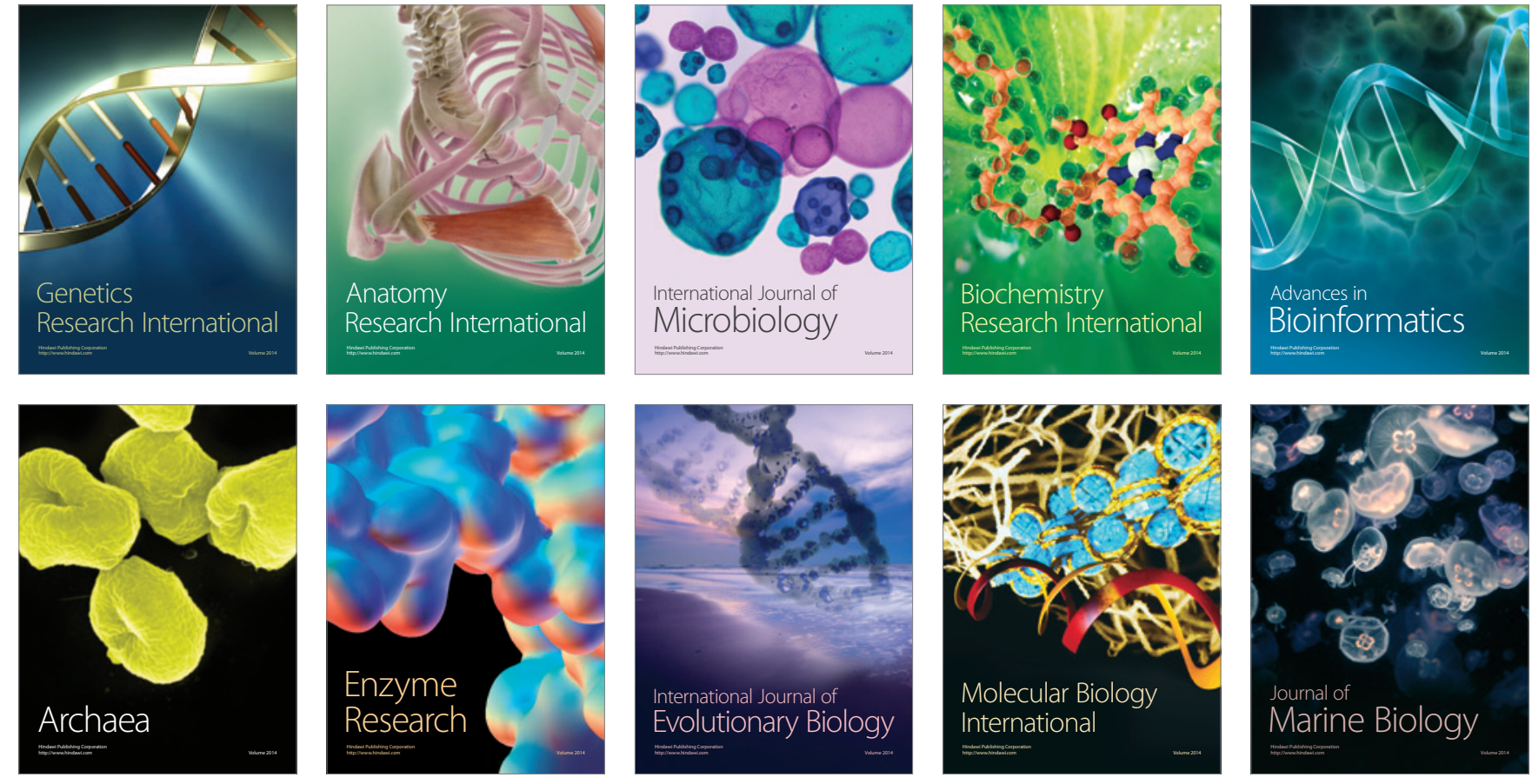